\title{
Influence of Different Surface Treatments on the Shear Bond Strength of Resin Cement to Base Metal Alloys
}

\author{
Farkhondeh Raeisosadat' ${ }^{1}$, Rahab Ghoveizi ${ }^{2 *}$, Solmaz Eskandarion ${ }^{3}$, Elaheh Beyabanaki ${ }^{2}$, Sara Tavakolizadeh ${ }^{2}$ \\ 'Department of Operative Dentistry, School of Dentistry, Shahid Beheshti University of Medical Sciences, Tehran, Iran \\ ${ }^{2}$ Department of Prosthodontics, School of Dentistry, Shahid Beheshti University of Medical Sciences, Tehran, Iran \\ ${ }^{3}$ Dental Material Research Center, Tehran Medical Sciences, Islamic Azad university, Tehran, Iran
}

\author{
*Correspondence to \\ Rahab Ghoveizi, \\ Assistant Professor, Department \\ of Prosthodontics, School of \\ Dentistry Shahid Beheshti \\ university of medical sciences, \\ Tehran, Iran. \\ Tel: +989122902849 \\ Fax: +9822403194 \\ Email: r.ghoveizi@gmail.com
}

Published online January 18 2020

\begin{abstract}
Introduction: This study aimed to investigate the effect of different surface treatments on the bond strength of resin cement to nickel-chrome $(\mathrm{Ni}-\mathrm{Cr})$ alloy.

Methods: Forty disk-shaped specimens of $\mathrm{Ni}-\mathrm{Cr}$ alloy were prepared and divided into 4 groups. In the first group, the specimens' surface was sandblasted with $50 \mu \mathrm{Al}_{2} \mathrm{O}_{3}$ particles. In the second group, the specimens were prepared with the Er:YAG laser. In the third group, the specimens were prepared using the Er:YAG laser after sandblasting. In the fourth group, the specimens' surface was covered with a thin layer of MKZ metal primer after sandblasting. Then the cylinders of composite resin were bonded to the treated metal surfaces using Panavia F2.0 resin cement. All of the samples were subjected to 2000 thermal cycles. The shear bond strength was tested using a universal testing machine at the crosshead speed of $0.5 \mathrm{~mm} / \mathrm{min}$. The failure mode was also observed by a stereomicroscope. Data were analyzed using the one-way ANOVA and the Tukey HSD test at a significance level of 0.05 .

Results: The shear bond strength from the highest to the lowest were as follows: the Er:YAG laser group, the sandblast and MKZ primer combination group, the sandblast group, and the sandblast and Er:YAG laser combination group. The mean differences of shear bond strength between the Er:YAG laser group and the sandblast group $(P=0.047)$ and also between the Er:YAG laser group and the sandblast and Er:YAG laser combination group $(P=0.015)$ were statistically significant. Conclusion: Among the different surface treatments employed in this study, Er:YAG laser treatment increased the shear bond strength between the metal alloy and the resin cement (Pavnavia F2). Keywords: Shear bond strength; Alloy primer; Er:YAG laser; Base metal alloys.
\end{abstract}

\section{Introduction}

Metal-ceramic restorations are used for various reasons, the most significant reason of which is the loss of tooth structure due to caries and trauma. ${ }^{1}$ In this regard, the adhesion of the metal to tooth structure is very important in terms of retention and long-term durability of the restoration. The role of adhesion is especially important when the retention of restoration is compromised because of over-tapering and inadequate height of the prepared tooth. ${ }^{2}$ Based on the type of surface treatment of both the tooth and the metal surface of the metal-ceramic restoration and also the chemical composition of the cement, adhesion may be achieved by the process of luting, chemical reaction, and/or micromechanical retention. ${ }^{1}$ Surface preparations increase the available surface for adhesion. ${ }^{3}$ Therefore, various surface preparations, including sandblasting, using metal primers, tin plating, silicoating, and laser treatments have been recommended on base metal alloys to increase the bond strength to resin cement.

Sandblasting is a low-cost surface preparation technique which improves the wettability of cement by the mechanical removal of surface debris. ${ }^{2}$ Studies have shown a higher bond strength of resin cement to the sandblasted base metal alloys. ${ }^{4-6}$

A metal primer is a bipolar molecule with the potential of bonding to a metal alloy and copolymerization with resin compounds. The MKZ primer is one of the new types of metal primers and very few studies have evaluated its effect on the shear bond strength. ${ }^{7,8}$

Laser radiation is another method for developing surface changes in metal alloys and dental ceramics, which can lead to stronger bonding. ${ }^{9,10}$ The main laser energy effect is through the conversion of the energy of light to heat. ${ }^{11,12}$ Lasers used to make surface changes in metal alloys include Nd:YAG, Er:YAG, and Er,Cr:YSGG. ${ }^{13,14}$ Several 
quantitative studies have explored the effect of Er:YAG on the modification of base metal alloy surfaces. ${ }^{9-14}$ The Er:YAG laser has also been suggested for other uses such as the removal of dentin caries and the preparation of cavity. ${ }^{15,16}$

The resin bond to metal alloys has significantly improved during the last decade. The polymerization of dual-cure resin cement can be initiated by light or chemical reaction. These two mechanisms are the basis of the extensive application of these luting materials as permanent types of cement for various types of restorations, including base metal restorations. In addition, these types of cement compared with other types of cement such as zinc phosphate and glass ionomer have a greater resistance to displacement. ${ }^{17}$

Apart from the bonding quality of resin cement, the type of surface treatment is an important factor in the shear bond strength. Considering the development of new materials and methods to improve this bonding, the purpose of this study was to investigate and compare the effect of different surface methods on the shear bond strength of resin cement to the nickel-chrome (Ni-Cr) alloys.

\section{Materials and Methods}

Forty disks of Ni-Cr alloy (Verabond, Alba Dent, USA) with a diameter of $6 \mathrm{~mm}$ and a thickness of $2 \mathrm{~mm}$ were prepared according to the manufacturer's instructions and then mounted in self-curing acrylic resin blocks (Acropars, Marlik, Tehran, Iran). All of the samples were polished with a 600 grit silicon carbide abrasive paper. The first group was sandblasted with $50 \mathrm{\mu} \mathrm{Al}_{2} \mathrm{O}_{3}$ particles applied vertically to the surface at a 3 bar pressure for 14 seconds at a distance of $10 \mathrm{~mm}$. They were then prepared with 96\% isopropyl alcohol (Sigma-Aldrich, St Louis, USA) for 3 minutes ultrasonically and air-dried for another 3 minutes. In the second group, the surface of the specimens was prepared using the Er:YAG laser with 1.5 $\mathrm{W}$, frequency of $10 \mathrm{~Hz}$, pulse duration of $300 \mu \mathrm{s}$, fluence of $500 \mathrm{~mJ}$, and spot size of $800 \mu \mathrm{m}$ (Doctor-smile LASER, Brendola, Italy). ${ }^{18}$ The specimens in the third group were first sandblasted and then cleaned in the same way as the first group. Afterwards, they were prepared using the Er:YAG laser. The specimens in the fourth group were covered with MKZ primer (Bedent, Senden, Germany) after being sandblasted with $50 \mu \mathrm{Al}_{2} \mathrm{O}_{3}$ particles. In each group, one specimen was randomly examined after surface preparation by scanning electron microscope (SEM) (JSM-5600 Scanning Microscope; JEOL Ltd, Tokyo, Japan) (Figure 1).

To examine the shear bond strength, plastic tubes with $3 \mathrm{~mm}$ in diameter and height were filled with light curing composite resin (Filtek Z250, 3M ESPE, USA) with shade $A 3 / 5$. Then both sides of the composite were light polymerized (Heliolux DLX; Ivoclar Vivadent, Schaan, Liechtenstein) at the minimum intensity of $600 \mathrm{~mW} /$

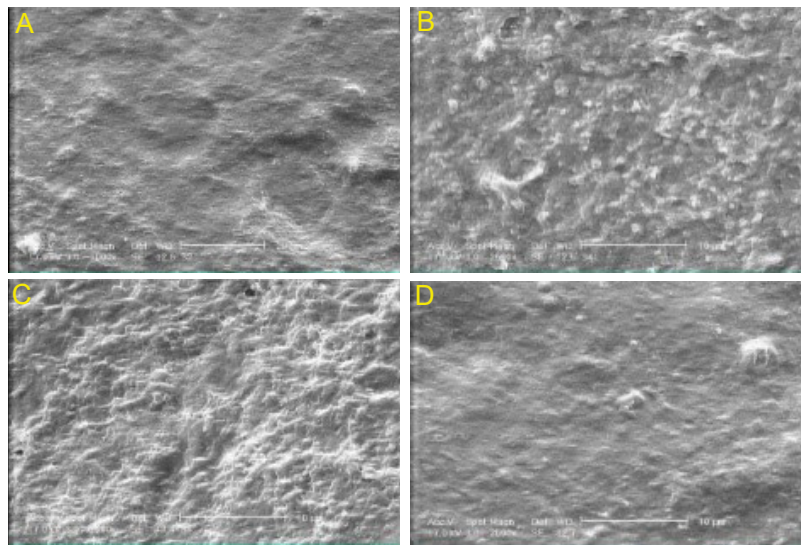

Figure 1. SEM images of base metal alloy surfaces prepared by (A) Sandblast, (B) Er:YAG laser, (C) Sandblast and Er:YAG laser combination, (D) Sandblast and MKZ primer combination.

$\mathrm{cm}^{2}$ for 40 seconds and bonded directly to the prepared substrate with Panavia F2.0 resin cement (Kuraray Noritake Dental Inc, Okayama, Japan). After 10 seconds of initial curing, the cement residue was removed and then Oxygen Blocking Gel (OxyGuard, Denmark) was used for 3 minutes. The final curing was done for 40 seconds from 4 directions. All of the specimens were put in distilled water at $37^{\circ} \mathrm{C}$ for 24 hours and then subjected to thermocycling with 2000 cycles between $5^{\circ} \mathrm{C}$ and $55^{\circ} \mathrm{C}$ and 20 seconds of exposure in each bath and transfer time of 10 seconds. After the specimens were dried at room temperature, their shear bond strength was tested using a universal testing machine (Santam, AST-20, Tehran, Iran) at a crosshead speed of $0.5 \mathrm{~mm} / \mathrm{min}$ until failure happened.

The failure mode was also detected by a stereomicroscope (Nikon SMZ800) with a magnification of 40x. The type of failure was divided into 2 categories of adhesive failure in the interface of metal-resin and mixed failure (the presence of cement residues or cement and composite at the metal surface). The one-way ANOVA and Tukey HSD tests were used to evaluate and compare the shear bond strength between the groups $(\alpha=0.05)$.

\section{Results}

Bond strength in $\mathrm{MPa}$ (mean $\pm \mathrm{SD}$ ) for all groups is presented in Table 1. According to the results, the highest shear bond strength was seen in the Er:YAG laser group. Then the sandblast and MKZ primer combination group, the sandblast group, and the sandblast and Er:YAG laser combination group showed the next highest shear bond strengths respectively.

The pairwise comparison between the groups using the Tukey HSD test showed that the mean shear bond strength was statistically significant only between the Er:YAG laser group and the sandblast group $(P=0.047)$, and also between the Er:YAG laser group and the sandblast and Er:YAG laser combination group $(P=0.015)$ (Table 2). 
Table 1. Bond Strength of Resin Cement to Base Metal Alloy After 4 Surface Treatments According to One-Way ANOVA With Significance Level of 0.015 Between the Groups

\begin{tabular}{lccc}
\hline Surface Treatments & $\begin{array}{c}\text { Mean } \pm \text { SD } \\
(\mathbf{M P a})\end{array}$ & Min & Max \\
\hline Sandblast & $17.40 \pm 0.95$ & 15.98 & 18.97 \\
Er:YAG laser & $19.93 \pm 2.47$ & 17.09 & 24.47 \\
Sandblast and Er:YAG laser combination & $16.96 \pm 2.10$ & 13.29 & 19.81 \\
Sandblast and MKZ primer combination & $18.46 \pm 1.98$ & 14.93 & 21.55 \\
\hline
\end{tabular}

The results of the failure mode of the specimens by the stereomicroscope showed that most failures in the Er:YAG laser group and the MKZ primer and sandblast combination group were of the mixed type. However, the failure type of the sandblast and Er:YAG laser combination group and the sandblast group was of adhesive nature (Table 3).

According to SEM images, the $\mathrm{Ni}-\mathrm{Cr}$ alloy surface showed morphological changes in all groups. Cluster views of asymmetric melting of the surface were observed for specimens prepared with the Er:YAG laser, which could justify a higher bond strength in this group.

\section{Discussion}

This study was conducted to find the best surface preparation method for resin cement bonding to $\mathrm{Ni}-\mathrm{Cr}$ alloy as a commonly used metal alloy in metal-ceramic restorations. One of the surface preparation methods used in this study was sandblasting. This method causes complex morphological changes in metal surfaces and also the accumulation of certain metal elements $\left(\mathrm{Al}_{2} \mathrm{O}_{3}\right)$ on the surface of the metal. ${ }^{19}$ In addition, sandblasting can improve the cement wettability through the mechanical removal of debris. This method has less technical sensitivity and lower cost than other metal surface treatments. ${ }^{20}$ Since many studies have shown a high bond strength of Panavia F2.0 resin cement to the sandblasted base metal alloys, ${ }^{4-6,21}$ all the specimens except the laser group were sandblasted before bonding. According to the findings of this study, the sandblasted metal alloy surfaces showed good bonding to Panavia F2.0 resin cement, although it was significantly less than that of the laser group $(P=0.047)$.

Since it has been proved that there is no significant difference between the Er:YAG laser and the Nd:YAG laser for surface treatment on the bond strength of resin cement, ${ }^{14,22}$ the Er:YAG was used in this study due to its availability. Based on the outcomes of this study, the highest bond strength was reported in the Er:YAG laser group, which can be associated with the surface roughness that the laser develops on the surface of the alloy. ${ }^{13,14}$

By examining the images of the SEM on the surfaces exposed to Er:YAG laser operations, cluster views were observed due to the asymmetric melting of the surface, which could justify the higher bond strength found in this group. The Er:YAG laser is also capable of removing the particles by micro-explosion and vaporization, a process which is referred to as ablation. ${ }^{23}$

However, there was a significant decrease in the bond strength when the Er:YAG laser was applied after sandblasting. The use of the Er:YAG laser after sandblasting seemed to lead to excessive surface changes and degradation of microscopic structures on the surface. Akyil et $\mathrm{al}^{24}$ concluded that the Er:YAG laser alone increased surface texture in zirconia ceramics, but laser application after sandblasting might degrade this structure. This also might be the reason for the lower shear bond strength observed in the sandblast and Er:YAG laser combination group.

This study also revealed that the mean shear bond strength in the sandblast and MKZ primer combination group was the highest after the laser group, while this difference was not statistically significant. This finding was similar to the results of Shafiei et $\mathrm{al}^{4}$ that found

Table 2. Pairwise Comparison of Different Surface Treatment Methods

\begin{tabular}{|c|c|c|c|c|c|c|}
\hline \multirow{2}{*}{ Group } & \multirow{2}{*}{ Group } & \multirow{2}{*}{ Mean Difference } & \multirow{2}{*}{ SE } & \multirow{2}{*}{$P$ Value } & \multicolumn{2}{|c|}{ 95\% Confidence Interval } \\
\hline & & & & & Lower Bound & Upper Bound \\
\hline Sandblast & Er:YAG laser & $-2.53222^{*}$ & 0.92493 & 0.047 & -5.0382 & -0.0262 \\
\hline Sandblast & Sandblast and Er:YAG laser & 0.43111 & 0.92493 & 0.966 & -2.0749 & 2.9371 \\
\hline Sandblast & Sandblast and MKZ primer & -1.06889 & 0.92493 & 0.658 & -3.5749 & 1.4371 \\
\hline Er:YAG laser & Sandblast and Er:YAG laser & $2.96333^{*}$ & 0.92493 & 0.015 & 0.4574 & 5.4693 \\
\hline Er:YAG laser & Sandblast and MKZ primer & 1.46333 & 0.92493 & 0.403 & -1.0426 & 3.9693 \\
\hline Sandblast and laser & Sandblast and MKZ primer & -1.5 & 0.92493 & 0.381 & -4.006 & 1.006 \\
\hline
\end{tabular}

* Significance level is $<0.05$

Table 3. Failure Mode Observed Under the Stereomicroscope

\begin{tabular}{lcccc}
\hline \multirow{2}{*}{ Failure mode } & \multicolumn{3}{c}{ Surface Treatment } \\
\cline { 2 - 5 } & Sandblast & Er:YAG Laser & Sandblast and Er:YAG Laser Combination & Sandblast and MKZ Primer Combination \\
\hline Adhesive & 5 & 3 & 5 & 2 \\
Mixed & 4 & 6 & 4 & 7 \\
\hline
\end{tabular}


higher bond strength for the combination of sandblasting and metal primers. However, they did not include laser treatment in their study. The MKZ metal primer is a bipolar molecule that is capable of bonding to a metal alloy and also has the potential for copolymerization with the resin compounds. ${ }^{7,8}$ This primer can also increase the wettability of the substrate surface. ${ }^{25}$ Moreover, most failures of this group and the Er:YAG laser group observed under the stereomicroscope were of the mixed type, which indicated a stronger bond in these 2 groups.

One of the limitations of this study was the lack of cyclic loading to examine the long-term durability of bond strength of the surfaces subjected to various surface treatments. Furthermore, evaluating shear bond strength to dentin under clinical situations is recommended.

\section{Ethical Considerations}

This research is an in-vitro study, so there are no ethical issues to be stated.

\section{Conflict of Interests}

The authors declare no conflict of interest.

\section{References}

1. Piwowarczyk A, Lauer HC, Sorensen JA. In vitro Shear bond strength of cementing agents to fixed prosthodontic restorative materials. J Prosthet Dent. 2004; 92(3):26573. doi: 10.1016/j.prosdent.2004.06.027.

2. Abreu A, Loza MA, Elias A, Mukhopadhyay S, Looney S, Rueggeberg FA. Tensile bond strength of an adhesive resin cement to different alloys having various surface treatments. J Prosthet Dent. 2009;101(2):107-18. doi: 10.1016/S0022-3913(09)60004-4.

3. Gorler O, Ozdemir AK. Bonding strength of ceromer with direct laser sintered, Ni-Cr-based, and $\mathrm{ZrO} 2$ metal infrastructures after Er:YAG, Nd:YAG, and Ho:YAG laser surface treatments - a comparative in vitro study. Photomed Laser Surg. 2016;34(8):355-62. doi: 10.1089/ pho.2016.4129.

4. Shafiei F, Behroozibakhsh M, Abbasian A, Shahnavazi $\mathrm{S}$. Bond strength of self-adhesive resin cement to base metal alloys having different surface treatments. Dent Res J (Isfahan). 2018;15(1):63-70. doi: 10.4103/17353327.223610.

5. Pegoraro LF, Barrack G. A Comparison of bond strength of adhesive cast restorations using different designs, bonding agents and luting agents. J Prosthet Dent. 1987;57(2):133-8. doi: 10.1016/0022-3913(87)90134-x.

6. Al-Helou H, Swed E. Effect of metal type and surface treatment on shear bond strength of resin cement (in vitro study). J Indian Prosthodont Soc. 2016;16(1):49-52. doi: 10.4103/0972-4052.164882.

7. Sanohkan S, Urapepon S, Harnirattisai C, Sirisinha C, Sunintaboon P. Shear bond strength between autopolymerizing acrylic resin and Co-Cr alloy using different primers. Dent Mater J. 2012;31(5):765-71. doi: 10.4012/dmj.2012-051.

8. Fonseca RG, Martins SB, de Oliveira Abi-Rached F,
Dos Santos Cruz CA. Effect of different airborneparticle abrasion/bonding agent combinations on the bond strength of a resin cement to a base metal alloy. J Prosthet Dent. 2012;108(5):316-23. doi: 10.1016/S00223913(12)60185-1.

9. Dede DÖ, Yenisey M, Rona N, Öngöz Dede F. Effects of Laser Treatment on the Bond Strength of Differently Sintered Zirconia Ceramics. Photomed Laser Surg. 2016;34(7):276-83. doi: 10.1089/pho.2015.4064.

10. Çağlar I, Yanikoğlu N. The effect of sandblasting, Er:YAG laser, and heat treatment on the mechanical properties of different zirconia cores. Photomed Laser Surg. 2016;34(1):17-26. doi: 10.1089/pho.2015.3980.

11. Kurklu D, Yanikoglu N. SEM evaluation of surface morphologic analysis of restorative materials with three laser treatment. J Dent Lasers. 2013;7(1):9-16. doi: 10.4103/0976-2868.118414.

12. Oyar P. Laser sintering technology and balling phenomenon. Photomed Laser Surg. 2018;36(2):72-7. doi: 10.1089/pho.2017.4311.

13. Sadat Madani A, Astaneh PA, Shahabi S, Nakhaei MR, Bagheri HG, Chiniforush N. Influence of different power outputs of intraoral Nd:YAG laser on shear bond strength of a resin cement to nickel-chromium dental alloy. Lasers Med Sci. 2013;28(1):229-34. doi: 10.1007/ s10103-012-1095-2.

14. Kunt GE, Güler AU, Ceylan G, Duran I, Ozkan P, Kirtiloğlu T. Effects of Er:YAG laser treatments on surface roughness of base metal alloys. Lasers Med Sci. 2012;27(1):47-51. doi: 10.1007/s10103-010-0844-3.

15. Delmé KI, De Moor RJ. Scanning electron microscopic evaluation of enamel and dentin surfaces after Er:YAG laser preparation and laser conditioning. Photomed Laser Surg. 2007; 25(5):393- 401. doi: 10.1089/ pho.2006.2069.

16. Shiu P, De Souza-Zaroni WC, de Eduardo CP, Youssef MN. Effect of feldspathic ceramic surface treatments on bond strength to resin cement. Photomed Laser Surg. 2007;25(4):291-6. doi: 10.1089/pho.2007.2018.

17. Ferracane JL, Stansbury JW, Burke FJ. Self-adhesive resin cements - chemistry, properties and clinical considerations.J Oral Rehabil.2011;38(4):295-314. doi: 10.1111/j.1365-2842.2010.02148.x.

18. Kunt GE, Güler AU, Ceylan G, Duran I, Ozkan P, Kirtiloğlu T. Effects of Er:YAG laser treatments on surface roughness of base metal alloys. Lasers Med Sci. 2012;27(1):47-51. doi: 10.1007/s10103-010-0844-3.

19. Kern M, Thompson VP. Sandblasting and silica-coating of dental alloys: volume loss, morphology and changes in the surface composition. Dent Mater. 1993;9(3):15161. doi: 10.1016/0109-5641(93)90113-5.

20. Abreu A, Loza MA, Elias A, Mukhopadhyay S, Rueggeberg FA. Effect of metal type and Surface treatment on in vitro tensile strength of copings cemented to minimally retentive preparations. J Prosthet Dent. 2007;98(3): 199-207. doi: 10.1016/S00223913(07)60056-0.

21. Raeisosadat F, Ghavam M, Hasani Tabatabaei M, Arami $\mathrm{S}$, Sedaghati M. Bond strength of resin cements to noble 
and base metal alloys with different surface treatments. $J$ Dent (Tehran). 2014;11(5):596-603.

22. da Silva Ferreira S, Hanashiro FS, de Souza-Zaroni WC, Turbino ML, Youssef MN. Influence of aluminum oxide sandblasting associated with Nd:YAG or Er:YAG lasers on shear bond strength of a feldspathic ceramic to resin cements. Photomed Laser Surg. 2010; 28(4):471-5. doi: 10.1089/pho.2009.2528.

23. Cavalcanti AN, Foxton RM, Watson TF, Oliveira MT, Giannini M, Marchi GM. Bond strength of resin cements to a zirconia ceramic with different surface treatments.
Oper Dent. 2009;34(3):280-7. doi: 10.2341/08-80.

24. Akyil MS, Uzun IH, Bayindir F. Bond strength of resin cement to yttrium-stabilized tetragonal zirconia ceramic treated with air abrasion, silica coating, and laser irradiation. Photomed Laser Surg. 2010;28(6):8018. doi: 10.1089/pho.2009.2697.

25. Atsu SS, Kilicarslan MA, Kucukesmen HC, Aka PS. Effect of zirconium-oxide ceramic surface treatments on the bond strength to adhesive resin. J Prosthet Dent. 2006;95(6):430-6. doi: 10.1016/j.prosdent.2006.03.016. 